\title{
Discovery of gamma-ray emission from the extragalactic pulsar wind nebula N157B with H.E.S.S. ${ }^{\star}$
}

\author{
H.E.S.S. Collaboration, A. Abramowski ${ }^{1}$, F. Acero ${ }^{2}$, F. Aharonian ${ }^{3,4,5}$, A. G. Akhperjanian ${ }^{6,5}$, G. Anton ${ }^{7}$, \\ S. Balenderan ${ }^{8}$, A. Balzer ${ }^{7}$, A. Barnacka ${ }^{9}, 10$, Y. Becherini ${ }^{11,12}$, J. Becker ${ }^{13}$, K. Bernlöhr ${ }^{3,14}$, E. Birsin ${ }^{14}$, J. Biteau ${ }^{12}$, \\ A. Bochow ${ }^{3}$, C. Boisson ${ }^{15}$, J. Bolmont ${ }^{16}$, P. Bordas ${ }^{17}$, J. Brucker ${ }^{7}$, F. Brun ${ }^{12}$, P. Brun ${ }^{10}$, T. Bulik ${ }^{18}$, S. Carrigan ${ }^{3}$, \\ S. Casanova ${ }^{19,3}$, M. Cerruti ${ }^{15}$, P. M. Chadwick ${ }^{8}$, A. Charbonnier ${ }^{16}$, R. C. G. Chaves ${ }^{10,3}$, A. Cheesebrough ${ }^{8}$, \\ G. Cologna ${ }^{20}$, J. Conrad $^{21}$, C. Couturier ${ }^{16}$, M. Dalton ${ }^{14,22,23}$, M. K. Daniel ${ }^{8}$, I. D. Davids ${ }^{24}$, B. Degrange ${ }^{12}$, C. Deil ${ }^{3}$, \\ H. J. Dickinson ${ }^{21}$, A. Djannati-Ataii ${ }^{11}$, W. Domainko ${ }^{3}$, L. O'C. Drury ${ }^{4}$, G. Dubus ${ }^{25}$, K. Dutson ${ }^{26}$, J. Dyks ${ }^{9}$, M. Dyrda ${ }^{27}$, \\ K. Egberts ${ }^{28}$, P. Eger $^{7}$, P. Espigat ${ }^{11}$, L. Fallon ${ }^{4}$, C. Farnier ${ }^{21}$, S. Fegan ${ }^{12}$, F. Feinstein ${ }^{2}$, M. V. Fernandes ${ }^{1}$, \\ D. Fernandez ${ }^{2}$, A. Fiasson ${ }^{29}$, G. Fontaine ${ }^{12}$, A. Förster ${ }^{3}$, M. Füßling ${ }^{14}$, M. Gajdus ${ }^{14}$, Y. A. Gallant ${ }^{2}$, T. Garrigoux ${ }^{16}$, \\ H. Gast ${ }^{3}$, L. Gérard ${ }^{11}$, B. Giebels ${ }^{12}$, J. F. Glicenstein ${ }^{10}$, B. Glück ${ }^{7}$, D. Göring ${ }^{7}$, M.-H. Grondin ${ }^{3,20}$, S. Häffner ${ }^{7}$, \\ J. D. Hague ${ }^{3}$, J. Hahn ${ }^{3}$, D. Hampf ${ }^{1}$, J. Harris ${ }^{8}$, M. Hauser ${ }^{20}$, S. Heinz ${ }^{7}$, G. Heinzelmann ${ }^{1}$, G. Henri' ${ }^{25}$, G. Hermann ${ }^{3}$, \\ A. Hillert ${ }^{3}$, J. A. Hinton ${ }^{26}$, W. Hofmann ${ }^{3}$, P. Hofverberg ${ }^{3}$, M. Holler ${ }^{7}$, D. Horns ${ }^{1}$, A. Jacholkowska ${ }^{16}$, O. C. de Jager ${ }^{19}$, \\ C. Jahn ${ }^{7}$, M. Jamrozy ${ }^{30}$, I. Jung ${ }^{7}$, M. A. Kastendieck ${ }^{1}$, K. Katarzyński ${ }^{31}$, U. Katz ${ }^{7}$, S. Kaufmann ${ }^{20}$, B. Khélifi ${ }^{12}$, \\ D. Klochkov ${ }^{17}$, W. Kluźniak ${ }^{9}$, T. Kneiske ${ }^{1}$, Nu. Komin ${ }^{29}$, K. Kosack ${ }^{10}$, R. Kossakowski ${ }^{29}$, F. Krayzel ${ }^{29}$, H. Laffon ${ }^{12}$, \\ G. Lamanna ${ }^{29}$, J.-P. Lenain ${ }^{20}$, D. Lennarz ${ }^{3}$, T. Lohse ${ }^{14}$, A. Lopatin ${ }^{7}$, C.-C. Lu$^{3}$, V. Marandon ${ }^{3}$, A. Marcowith ${ }^{2}$, \\ J. Masbou ${ }^{29}$, G. Maurin ${ }^{29}$, N. Maxted ${ }^{32}$, M. Mayer ${ }^{7}$, T. J. L. McComb ${ }^{8}$, M. C. Medina ${ }^{10}$, J. Méhault ${ }^{2,22,23}$, \\ U. Menzler ${ }^{13}$, R. Moderski ${ }^{9}$, M. Mohamed ${ }^{20}$, E. Moulin ${ }^{10}$, C. L. Naumann ${ }^{16}$, M. Naumann-Godo ${ }^{10}$, M. de Naurois ${ }^{12}$, \\ D. Nedbal ${ }^{33}$, N. Nguyen ${ }^{1}$, B. Nicholas ${ }^{32}$, J. Niemiec ${ }^{27}$, S. J. Nolan ${ }^{8}$, S. Ohm ${ }^{34,26,3}$, E. de Oña Wilhelmi ${ }^{3}$, B. Opitz ${ }^{1}$, \\ M. Ostrowski ${ }^{30}$, I. Oya ${ }^{14}$, M. Panter ${ }^{3}$, M. Paz Arribas ${ }^{14}$, N. W. Pekeur ${ }^{19}$, G. Pelletier ${ }^{25}$, J. Perez ${ }^{28}$, P.-O. Petrucci ${ }^{25}$, \\ B. Peyaud ${ }^{10}$, S. Pita ${ }^{11}$, G. Pühlhofer ${ }^{17}$, M. Punch ${ }^{11}$, A. Quirrenbach ${ }^{20}$, M. Raue ${ }^{1}$, A. Reimer ${ }^{28}$, O. Reimer ${ }^{28}$, \\ M. Renaud ${ }^{2}$, R. de los Reyes ${ }^{3}$, F. Rieger ${ }^{3}$, J. Ripken ${ }^{21}$, L. Rob $^{33}$, S. Rosier-Lees ${ }^{29}$, G. Rowell ${ }^{32}$, B. Rudak ${ }^{9}$, \\ C. B. Rulten ${ }^{8}$, V. Sahakian ${ }^{6,5}$, D. A. Sanchez ${ }^{3}$, A. Santangelo ${ }^{17}$, R. Schlickeiser ${ }^{13}$, A. Schulz ${ }^{7}$, U. Schwanke ${ }^{14}$, \\ S. Schwarzburg ${ }^{17}$, S. Schwemmer ${ }^{20}$, F. Sheidaei ${ }^{11,19}$, J. L. Skilton ${ }^{3}$, H. Sol ${ }^{15}$, G. Spengler ${ }^{14}$, Ł. Stawarz ${ }^{30}$, \\ R. Steenkamp ${ }^{24}$, C. Stegmann ${ }^{7}$, F. Stinzing ${ }^{7}$, K. Stycz ${ }^{7}$, I. Sushch ${ }^{14}$, A. Szostek ${ }^{30}$, J.-P. Tavernet ${ }^{16}$, R. Terrier ${ }^{11}$, \\ M. Tluczykont ${ }^{1}$, K. Valerius ${ }^{7}$, C. van Eldik ${ }^{7,3}$, G. Vasileiadis ${ }^{2}$, C. Venter ${ }^{19}$, A. Viana ${ }^{10}$, P. Vincent ${ }^{16}$, H. J. Völk ${ }^{3}$, \\ F. Volpe ${ }^{3}$, S. Vorobiov ${ }^{2}$, M. Vorster ${ }^{19}$, S. J. Wagner ${ }^{20}$, M. Ward ${ }^{8}$, R. White ${ }^{26}$, A. Wierzcholska ${ }^{30}$, M. Zacharias ${ }^{13}$, \\ A. Zajczyk ${ }^{9,2}$, A. A. Zdziarski ${ }^{9}$, A. Zech ${ }^{15}$, and H.-S. Zechlin ${ }^{1}$
}

(Affiliations can be found after the references)

Received 27 June 2012 / Accepted 6 August 2012

\section{ABSTRACT}

We present the significant detection of the first extragalactic pulsar wind nebula (PWN) detected in gamma rays, N 157B, located in the large Magellanic Cloud (LMC). Pulsars with high spin-down luminosity are found to power energised nebulae that emit gamma rays up to energies of several tens of TeV. N 157B is associated with PSR J0537-6910, which is the pulsar with the highest known spin-down luminosity. The High Energy Stereoscopic System telescope array observed this nebula on a yearly basis from 2004 to 2009 with a dead-time corrected exposure of $46 \mathrm{~h}$. The gamma-ray spectrum between $600 \mathrm{GeV}$ and $12 \mathrm{TeV}$ is well-described by a pure power-law with a photon index of $2.8 \pm 0.2_{\text {stat }} \pm 0.3_{\text {syst }}$ and a normalisation at $1 \mathrm{TeV}$ of $\left(8.2 \pm 0.8_{\text {stat }} \pm 2.5_{\text {syst }}\right) \times 10^{-13} \mathrm{~cm}^{-2} \mathrm{~s}^{-1} \mathrm{TeV}^{-1}$. A leptonic multi-wavelength model shows that an energy of about $4 \times 10^{49} \mathrm{erg}$ is stored in electrons and positrons. The apparent efficiency, which is the ratio of the TeV gamma-ray luminosity to the pulsar's spin-down luminosity, $0.08 \% \pm 0.01 \%$, is comparable to those of PWNe found in the Milky Way. The detection of a PWN at such a large distance is possible due to the pulsar's favourable spin-down luminosity and a bright infrared photon-field serving as an inverse-Compton-scattering target for accelerated leptons. By applying a calorimetric technique to these observations, the pulsar's birth period is estimated to be shorter than $10 \mathrm{~ms}$.

Key words. gamma rays: general - pulsars: individual: PSR J0537-6910 - ISM: supernova remnants - Magellanic Clouds

\section{Introduction}

* Data set is only available at the CDS via anonymous ftp to cdsarc.u-strasbg.fr (130.79.128.5) or via http://cdsarc.u-strasbg.fr/viz-bin/qcat?J/A+A/545/L2
In recent years, many Galactic pulsar wind nebulae (PWNe) have been discovered to be gamma-ray emitters (for a review, see e.g. de Oña Wilhelmi 2011). It was predicted by Aharonian et al. (1997) that the gamma-ray luminosity of these nebulae 

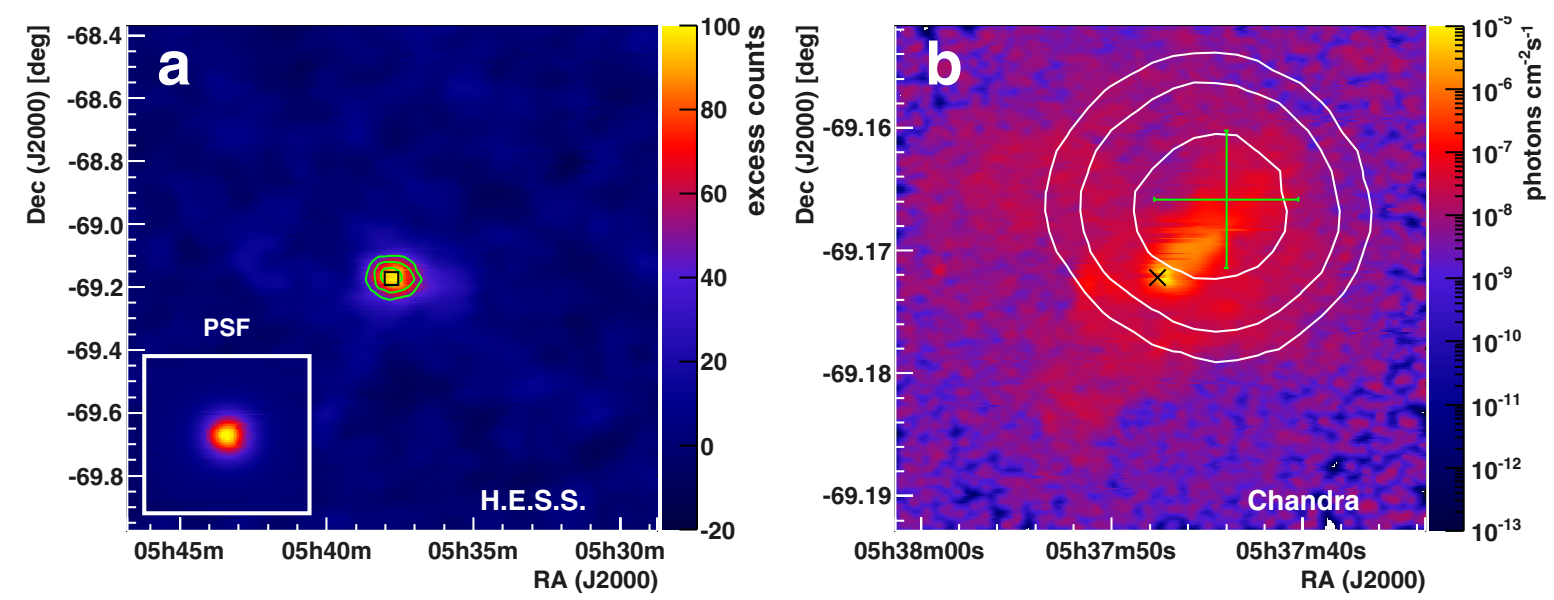

Fig. 1. Gamma-ray and X-ray view of N 157B. a) Image of the TeV gamma-ray excess obtained with H.E.S.S. The image was smoothed with the instrument's point-spread function, which is shown in the inset. The green contour lines denote 6,9 , and $12 \sigma$ statistical significances. The black box outlines the region shown in the X-ray image. b) Image of the X-ray flux in the energy band from $0.8 \mathrm{keV}$ to $8 \mathrm{keV}$ (Chandra ACIS-S, observation $\mathrm{n}^{\circ} .2783,48.2 \mathrm{ks}$ dead-time corrected exposure). The data were smoothed with a Gaussian of $\sigma=1.5^{\prime \prime}$ and the exposure map was computed with an assumed spectral index of 2.3, which is the average index of the nebula. The colour coding represents the X-ray flux in photons $\mathrm{cm}^{-2} \mathrm{~s}^{-1}$; for better visibility, it is clipped at $10^{-13}$ photons $\mathrm{cm}^{-2} \mathrm{~s}^{-1}$ at the lower end and saturates at $10^{-5}$ photons $\mathrm{cm}^{-2} \mathrm{~s}^{-1}$. The white contour lines denote regions of $68 \%, 95 \%$, and $99 \%$ confidence for the position of the gamma-ray source HESS J0537-691. The position of the pulsar PSR J0537-6910 is indicated by a black cross. The green cross marks the best-fit position and the systematic uncertainty in the pointing of the H.E.S.S. telescopes.

is connected to the spin-down power $\dot{E}$, i.e. the loss rate of rotational energy of the pulsar, and that pulsars with $\dot{E}>$ $10^{34-35}(d / 1 \mathrm{kpc})^{2} \mathrm{erg} \mathrm{s}^{-1}$ (with $d$ being the distance to the pulsar) power nebulae that are detectable in gamma rays. On the basis of the Galactic plane survey carried out by H.E.S.S. (Aharonian et al. 2005; Aharonian et al. 2006b; Gast et al. 2012), Carrigan et al. (2008) suggested that pulsars with a spin-down power of $\dot{E} \gtrsim 10^{34}(\mathrm{~d} / 1 \mathrm{kpc})^{2} \mathrm{erg} \mathrm{s}^{-1}$ may be correlated with nebulae detectable by H.E.S.S.

The most energetic pulsar known is PSR J0537-6910, with a spin-down power of $\dot{E}=4.9 \times 10^{38} \mathrm{erg} \mathrm{s}^{-1}$ (Marshall et al. 1998; Manchester et al. 2005). PSR J0537-6910 is also one the most distant pulsars known to date: located in the LMC, it has an estimated distance of $\left(48.1 \pm 2.3_{\text {stat }} \pm 2.9_{\text {syst }}\right) \mathrm{kpc}$ (Macri et al. 2006). This pulsar is the compact central object of the supernova remnant (SNR) N 157B (also called LHA 120-N 157B, SNR B0538-691, or NGC 2060). N 157B has been observed extensively in X-rays (Wang \& Gotthelf 1998; Wang et al. 2001; Chen et al. 2006). N 157B is a Crab-like SNR (Gotthelf \& Wang 1996): the emission from N 157B is dominated by synchrotron radiation from the $\mathrm{PWN}$, which shows a bar-like feature surrounding the pulsar representing the reverse shock of a toroidal wind from the pulsar as well as a long tail of diffuse emission of about $20^{\prime \prime} \times 30^{\prime \prime}$ in the north-west direction from the bar Wang et al.; Chen et al. (2001; 2006, see also Fig. 1b). Chen et al. (2006) show that faint thermal emission with a diameter of about $100^{\prime \prime}$ is observed from the supernova ejecta and, unlike shell-type or composite SNRs, no emission from the supernova forward shock is detected. The supernova ejecta apparently expand into the low-density interior of the superbubble formed by the stellar association LH 99 (Chen et al. 2006). The characteristic age of the pulsar is about 5000 years (Marshall et al. 1998), which is consistent with estimates of the SNR's age (Wang \& Gotthelf 1998). Large-scale diffuse emission from the general direction of N 157B was discovered with the Fermi satellite (Abdo et al. 2010), which was interpreted as emission from massive star-forming regions. No significant emission, in addition to the diffuse emission, has been detected from N 157B.
Despite its extreme distance, PSR J0537-6910 has a large enough spin-down flux of $\dot{E} / d^{2}=2 \times 10^{35} \mathrm{erg} \mathrm{s}^{-1} \mathrm{kpc}^{-2}$ to power a nebula detectable with H.E.S.S.

\section{H.E.S.S. observations and results}

The High Energy Stereoscopic System (H.E.S.S.; Aharonian et al. 2006a) is a system of four Imaging Cherenkov Telescopes, located in the Khomas Highland of Namibia at an altitude of $1800 \mathrm{~m}$. The H.E.S.S. telescope array's location in the southern hemisphere is ideal for observing the Magellanic Clouds. Furthermore, H.E.S.S. is sensitive to gamma rays at energies above $100 \mathrm{GeV}$ up to several $10 \mathrm{TeV}$. The arrival direction of individual gamma rays can be reconstructed with an angular resolution of higher than $0.1^{\circ}$, and their energy is estimated with an relative uncertainty of $15 \%$.

The region containing N 157B was observed with H.E.S.S., the data presented having been recorded from 2004 to 2009, with a dead-time corrected exposure of $46 \mathrm{~h}$ after data quality selection. The observations were carried out at large zenith angles (with a mean of $47^{\circ}$ ) leading to an elevated energy threshold of about $600 \mathrm{GeV}$. The data were analysed using Model analysis with standard cuts (de Naurois \& Rolland 2009), where the camera images are compared with simulations using a log-likelihood minimisation. The remaining background was estimated from both rings around each sky position to generate the gamma-ray image and spatial analysis (ring background, Berge et al. 2007) and test regions with similar offsets from the camera centre for the spectral analysis (reflected background, Berge et al. 2007).

Figure 1a shows a gamma-ray excess image of N 157B. The image was smoothed with the H.E.S.S. point-spread function, where $68 \%$ of the events were contained in a circle with a radius of $0.06^{\circ}$. The significance was calculated from the counts in a circular integration region with a radius of $0.06^{\circ}$ around each sky bin. Fitting a point-like source folded with the instrument's point spread function results in a best-fit position of the source of RA $=5^{\mathrm{h}} 37^{\mathrm{m}} 44^{\mathrm{s}}$, Dec $=-69^{\circ} 9^{\prime} 57^{\prime \prime}$, equinox J2000, with a statistical uncertainty of $\pm 11^{\prime \prime}$ in each direction; the source is hence 
labelled HESS J0537-691. With the H.E.S.S. standard pointing correction, point-like sources can be localised with a systematic uncertainty of $20^{\prime \prime}$ per axis (Acero et al. 2010). Figure $1 \mathrm{~b}$ shows an X-ray image of the supernova remnant and its central PWN with overlaid confidence contours of the gamma-ray source position. The best-fit position is consistent with the pulsar position; the slight offset from the pulsar along the tail of the PWN is not significant $(1.3 \sigma$ of the combined statistical and systematic error).

In a circular region with a radius of $0.1^{\circ}$ around the pulsar position, 395 gamma-ray candidate events were found. A total number of 3152 events were found in a background region with an area that is larger by a factor of 18.62 . The corresponding gamma-ray excess is 226 events with a statistical significance of $14 \sigma$ (calculated using formula (17) of Li \& Ma 1983). The photon spectrum between $600 \mathrm{GeV}$ and $12 \mathrm{TeV}$ of the gamma-ray excess can be described by a pure power-law, $\mathrm{d} N / \mathrm{d} E=\Phi_{0}(E / 1 \mathrm{TeV})^{-\Gamma}$, with a normalisation at $1 \mathrm{TeV}$ of $\Phi_{0}=\left(8.2 \pm 0.8_{\text {stat }}\right) \times 10^{-13} \mathrm{~cm}^{-2} \mathrm{~s}^{-1} \mathrm{TeV}^{-1}$ and a photon index of $\Gamma=2.8 \pm 0.2_{\text {stat }}$, which corresponds to an energy flux between $1 \mathrm{TeV}$ and $10 \mathrm{TeV}$ of $(1.4 \pm 0.1) \times 10^{-12} \mathrm{erg} \mathrm{cm}^{-2} \mathrm{~s}^{-1}$ or $2 \%$ of the energy flux of the Crab Nebula (Aharonian et al. 2006a). The gamma-ray luminosity between $1 \mathrm{TeV}$ and $10 \mathrm{TeV}$ for a distance of $48 d_{48} \mathrm{kpc}$ is $(3.9 \pm 0.3) \times 10^{35} d_{48}^{2} \mathrm{erg} \mathrm{s}^{-1}$. This result was derived from data accumulated over 4 years during the summer rainy seasons with varying atmospheric conditions. From the analysis of different subsets of the data, a systematic uncertainty of $30 \%$ in the flux and 0.3 in the photon index is estimated $^{1}$.

\section{Discussion}

The observed gamma-ray luminosity between $1 \mathrm{TeV}$ and $10 \mathrm{TeV}$ of the PWN corresponds to $(0.08 \pm 0.01) d_{48}^{2} \%$ of the spin-down power of the pulsar, a value typical of young PWNe (for a review see e.g. de Oña Wilhelmi 2011). Pulsar wind nebulae are very efficient in producing $\mathrm{TeV}$ gamma rays via the inverse Compton (IC) up-scattering of lower-energy photons by relativistic electrons ${ }^{2}$. This makes them not only the most abundant source type among the TeV emitters in the Milky Way, but also with HESS J0537-691 the first extragalactic TeV source that is unrelated to a galaxy or an active galactic nucleus. N 157B can be detected at such a large distance not only because of the high spin-down power of the pulsar but also because of the strong infrared-photon fields from nearby sources serving as additional targets for the IC scattering.

Figure 2 shows the spectral energy distribution (SED) of $\mathrm{N}$ 157B. It exhibits synchrotron emission that has been detected at radio wavelengths (see Lazendic et al. 2000, and references therein) and in X-rays (Chen et al. 2006; Dennerl et al. 2001), as well as $\mathrm{TeV}$ gamma-ray emission from IC scattering. In addition to the cosmic microwave background (CMB), far-infrared photons from the OB association LH 99 and the nearby star-forming region 30 Doradus are important targets for IC scattering. Using observations from Spitzer (Indebetouw et al. 2009), the infrared photon fields are modelled as black-body radiation with a temperature of $80 \mathrm{~K}$ and an energy density of $8.9 \mathrm{eV} \mathrm{cm}^{-3}$ for LH 99 , and a temperature of $88 \mathrm{~K}$ and an energy density of $2.7 \mathrm{eV} \mathrm{cm}^{-3}$ for 30 Doradus. These are only upper limits to the infrared fields, as the (unprojected) distances of N 157B to these objects are unknown.

\footnotetext{
1 These uncertainties are specific to this data set and should not be used for any other H.E.S.S. result.

2 The term electrons refers to electrons and positrons.
}

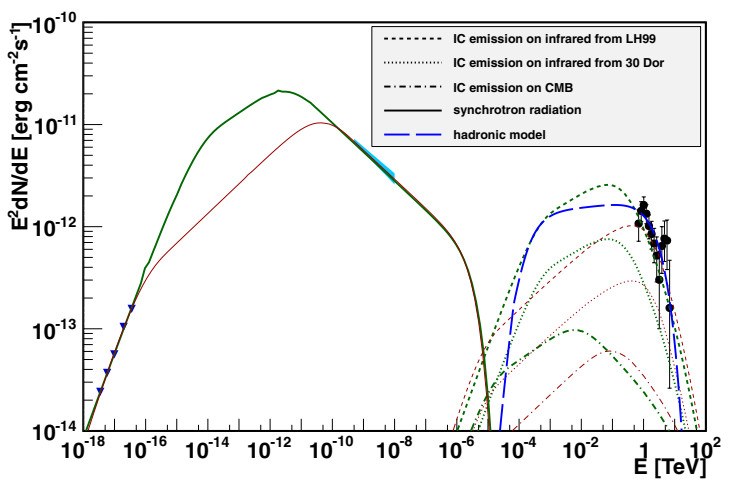

Fig. 2. Spectral energy distribution of N 157B. The data shown are radio emission (Lazendic et al. 2000, blue triangles), non-thermal X-rays (Chen et al. 2006, blue band), and TeV gamma rays (H.E.S.S., this work, black circles). The green, thick lines represent the model with a cooling break depending on the magnetic field and the age of the remnant. The red, thin lines denote the conservative model that minimises the energy content of electrons. The blue, long-dashed line represents a hadronic scenario.

There is no evidence of extended emission beyond the angular resolution of H.E.S.S., thus the synchrotron and IC emission regions cannot be separated spatially. Hence, a simple one-zone model is assumed, where only a single electron population is responsible for both the synchrotron and IC emission. An electron spectrum following a broken power-law with two breaks and covering the energy band from $1 \mathrm{eV}$ to $10^{15} \mathrm{eV}$ is adopted. The low energy break is an intrinsic break of the injection spectrum proposed by Venter \& de Jager (2007); the high energy break arises from the cooling of the particles. It is assumed that the cooling break appears at the energy where the synchrotron loss time (which depends on the magnetic field) is equal to the age of the remnant (which is assumed to be 5000 years). A lowenergy spectral index of 1.33 is used to reproduce the radio spectrum, and a spectral index of 3.60 above the cooling break is consistent with both the X-ray and $\mathrm{TeV}$ data. Assuming that the cooling steepens the spectral index by one (Kardashev 1962), the uncooled high-energy spectral index is 2.60. This model, which is represented by the green lines in Fig. 2, requires an intrinsic break at $54 \mathrm{GeV}$ and a magnetic field of $41 \mu \mathrm{G}$, the corresponding cooling break being at $1.1 \mathrm{TeV}$. Assuming a distance of $48 \mathrm{kpc}$, the total energy $W_{\text {tot }}$ stored in electrons is $4 \times 10^{49} \mathrm{erg}$. In a second, more conservative model, any assumptions about the age and cooling of the particles are abandoned to minimise the total energy stored in electrons. The intrinsic break is set to $7 \mathrm{GeV}$, the lowest energy still being compatible with the radio data, and the cooling break is set to $4.22 \mathrm{TeV}$, the highest energy being compatible with the $\mathrm{TeV}$ data points. These prerequisites require an uncooled high-energy spectral index of 2.57 and a low-energy spectral index of 0.93 to reproduce the radio data. This model is represented by the red lines in Fig. 2; it requires a magnetic field of $47 \mu \mathrm{G}$ and the total energy is only $50 \%$ of the energy in the first model. The uncertainty in the distance measurement adds an error of $15 \%$ to the estimate of $W_{\text {tot }}$. Major uncertainties in the estimation of $W_{\text {tot }}$ lie in the uncertainties in the gamma-ray spectrum. Varying the TeV flux by $30 \%$ while fixing the radio and X-ray points changes the total energy by $25 \%$. The total energy content $W_{\text {tot }}$ cannot be determined from the radio and X-ray data alone, since the strength of the synchrotron emission is governed by the a priori unknown magnetic field. Using the observed $\mathrm{TeV}$ spectrum presented here, it is possible to derive the magnetic field in the PWN and thus the energy content in electrons in the PWN. In a hadronic scenario, gamma-ray 
emission is produced in the decay of $\pi^{0}$ mesons produced by inelastic interactions of accelerated protons with ambient material. The observed $\mathrm{TeV}$ emission can be described by the emission of a proton population following a power-law with an index 2 and an exponential cut-off at $23 \mathrm{TeV}$ (blue, long-dashed line in Fig. 2). The total energy in this proton population is $W_{\text {had }}=1.8 \times 10^{52}\left(\mathrm{n} / \mathrm{cm}^{-3}\right)^{-1} d_{48}^{2}$ erg. An ambient density of at least $100 \mathrm{~cm}^{-3}$ would be necessary to produce this emission by a single supernova. That the SNR is expected to expand into the low-density interior of a superbubble makes this scenario unlikely.

As proposed by de Jager (2008), $W_{\text {tot }}$ can be used to estimate the birth period of the pulsar. PSR J0537-6910 is relatively young, its characteristic age being shorter than the cooling time of most of the electrons. Particles have survived since the earliest epoch when the pulsar's spin was close to its birth period. Therefore, $W_{\text {tot }}$ can be related to the pulsar's birth and current period by the calorimetric expression

$$
\begin{aligned}
W_{\mathrm{tot}} & =\epsilon \eta\left(E_{\mathrm{rot}, 0}-E_{\mathrm{rot}}\right) \\
& =\epsilon \eta \frac{1}{2} I\left(\left(\frac{2 \pi}{P_{0}}\right)^{2}-\left(\frac{2 \pi}{P}\right)^{2}\right) \\
& =2 \times 10^{50} \epsilon \eta \frac{I}{10^{45} \mathrm{~g} \mathrm{~cm}^{2}}\left(\left(\frac{10 \mathrm{~ms}}{P_{0}}\right)^{2}-\left(\frac{10 \mathrm{~ms}}{P}\right)^{2}\right) \mathrm{erg},
\end{aligned}
$$

where $P_{0}$ is the birth period of the pulsar, $P$ is the current period of $16.1 \mathrm{~ms}$ (Marshall et al. 1998), $\eta$ denotes the conversion efficiency of spin-down power into accelerated electrons, and $\epsilon$ is the relative average energy-loss rate, which takes into account the energy already radiated by the particles during earlier epochs and adiabatic losses of the energy. For the pulsar's moment of inertia, the canonical value of $I=10^{45} \mathrm{~g} \mathrm{~cm}^{2}$ is adopted (Lattimer \& Prakash 2001); the moment of inertia could be higher owing to variations in the pulsar mass between 1.4 and $2.5 M_{\odot}$ as proposed by Belczynski \& Taam (2008). Models indicate that $\eta$ can be as low as 0.3 (Schöck et al. 2010, for MSH 15-52) or as high as 0.7 (de Jager et al. 2008, for G21.5-0.9). Magnetohydrodynamical simulations (de Jager et al. 2009) show that the adiabatic energy loss is around $\epsilon \sim 0.5$. Further radiation losses during earlier epochs of the nebula reduce $\epsilon$ to less than 0.5 . Choosing 0.7 and 0.5 for $\eta$ and $\epsilon$, respectively, shows that the birth period must have been shorter than $10 \mathrm{~ms}$. Using the conservative model, the birth period increases by $23 \%$. This result is consistent with earlier estimations of the pulsar's birth period, Marshall et al. (1998) estimated the birth period by comparing the pulsar's characteristic age with the age of the SNR for different braking indices, for SNR ages of more than 4000 years the birth period is shorter than $10 \mathrm{~ms}$ for a large range of braking indices. From the extrapolation of glitch data, Marshall et al. (2004) found that 5000 years ago the pulsar period was $11 \mathrm{~ms}$. In the present paper, for the first time the birth period of a pulsar is obtained directly using a calorimetric technique, which depends on neither the glitch history nor the braking index and is - for the conservative model - completely independent of the age of the remnant.

This result confirms that PSR J0537-6910 has, with a birth period of less than about $10 \mathrm{~ms}$, the shortest birth period ever derived for a pulsar. These short rotation periods are only known for millisecond pulsars that have been spun up after their birth by a companion star. Simulations show that pulsar birth periods can be related to some parameters of the progenitor stars. Heger et al. (2005) show that more massive progenitor stars produce heavier and more rapidly rotating pulsars: stars of 15, 20 and $35 M_{\odot}$ are required to produce pulsars with $11 \mathrm{~ms}, 7 \mathrm{~ms}$ and $3 \mathrm{~ms}$, respectively. This is consistent with an earlier estimate of the PSR J0537-6910 progenitor mass of 20-35 $M_{\odot}$ based on the comparison of the observed metal abundances in the supernova ejecta of SNR N 157B with supernova models (Chen et al. 2006). Such massive stars are close to the threshold for the formation of black holes in the supernova explosion. Fryer (1999) show, for instance, that $25 M_{\odot}$ is roughly the limit for black hole formation. On the other hand, Ott et al. (2006) show that the pulsar's birth period is rather unrelated to the progenitor's mass but roughly linearly dependent on the initial central iron-core spin. Birth periods of less than $10 \mathrm{~ms}$ require initial iron core periods of less than about $8 \mathrm{~s}$. A very massive and/or rapidly spinning progenitor star therefore appears to be required to produce a neutron star with a birth period as short as $10 \mathrm{~ms}$.

Alternatively, the pulsar could be part of a binary system that has been spun up by its companion star, a scenario typically assumed for millisecond pulsars. A very massive and rapidly rotating star at a distance of about $0.8^{\prime}$ from PSR J0537-6910 has been identified (Dufton et al. 2011). It was proposed that both objects were part of a binary system where mass was transferred from the pulsar's progenitor to its companion and that both stars experienced radial velocity kicks in the supernova explosion. Nonetheless, in this scenario, the general picture of a very massive star producing a rapidly spinning neutron star remains unchanged.

\section{Conclusions}

Our principal conclusions are as follows:

1. Gamma-ray emission from the PWN in N157B was discovered with H.E.S.S. observations. The energy flux between $1 \mathrm{TeV}$ and $10 \mathrm{TeV}$ is $(1.4 \pm 0.1) \times 10^{-12} \mathrm{erg} \mathrm{cm}^{-2} \mathrm{~s}^{-1}$. Located in the LMC at a distance of $48 \mathrm{kpc}$, this is the most distant PWN ever detected in gamma rays and is the first individual stellar extragalactic TeV gamma-ray source. The PWN is powered by the most energetic pulsar known: PSR J0537-6910.

2. The TeV photon spectrum, in connection with radio and $\mathrm{X}$-ray measurements, can be described with a one-zone leptonic model. From this model, the total energy stored in electrons in the nebula can be estimated to be $4 \times 10^{49} \mathrm{erg}$.

3. For the pulsar to provide this energy from its rotational energy loss, the pulsar's birth period must have been shorter than about $10 \mathrm{~ms}$. This is the shortest birth period ever inferred for a pulsar. In an alternative scenario, the pulsar might have been spun up by a companion star in a binary system.

4. Assuming a direct connection between the pulsar's birth period and the mass of the progenitor star, the progenitor must have had a mass of at least $15 M_{\odot}$. This is close to the limit for black hole formation. The pulsar PSR J0537-6910 is therefore at the upper mass limit for neutron star production.

Acknowledgements. The support of the Namibian authorities and of the University of Namibia in facilitating the construction and operation of H.E.S.S. is gratefully acknowledged, as is the support by the German Ministry for Education and Research (BMBF), the Max Planck Society, the German Research Foundation (DFG), the French Ministry for Research, the CNRS-IN2P3 and the Astroparticle Interdisciplinary Programme of the CNRS, the UK Science and Technology Facilities Council (STFC), the IPNP of the Charles University, the Czech Science Foundation, the Polish Ministry of Science and Higher Education, the South African Department of Science and Technology and National Research Foundation, and by the University of Namibia. We appreciate the excellent work of the technical support staff in Berlin, Durham, Hamburg, Heidelberg, Palaiseau, Paris, Saclay, and in Namibia in the construction and operation of the equipment. 


\section{References}

Abdo, A. A., Ackermann, M., Ajello, M., et al. 2010, A\&A, 512, A7

Acero, F., Aharonian, F., Akhperjanian, A. G., et al. (HESS collaboration) 2010, MNRAS, 402, 1877

Aharonian, F. A., Atoyan, A. M., \& Kifune, T. 1997, MNRAS, 291, 162

Aharonian, F., Akhperjanian, A. G., Aye, K.-M., et al. (HESS collaboration) 2005, Science, 307, 1938

Aharonian, F., Akhperjanian, A. G., Bazer-Bachi, A. R., et al. (HESS collaboration) 2006a, A\&A, 457, 899

Aharonian, F., Akhperjanian, A. G., Bazer-Bachi, A. R., et al. (HESS collaboration) 2006b, ApJ, 636, 777

Belczynski, K., \& Taam, R. E. 2008, ApJ, 685, 400

Berge, D., Funk, S., \& Hinton, J. 2007, A\&A, 466, 1219

Carrigan, S., Hinton, J. A., Hofmann, W., et al. 2008, in International Cosmic Ray Conference, 2, 659

Chen, Y., Wang, Q. D., Gotthelf, E. V., et al. 2006, ApJ, 651, 237

de Jager, O. C. 2008, ApJ, 678, L113

de Jager, O. C., Ferreira, S. E. S., \& Djannati-Ataï, A. 2008, in AIP Conf. Ser. 1085, eds. F. A. Aharonian, W. Hofmann, \& F. Rieger, 199

de Jager, O. C., Ferreira, S. E. S., Djannati-Ataï, A., et al. 2009 [arXiv: 0906.2644]

de Naurois, M., \& Rolland, L. 2009, Astropart. Phys., 32, 231

de Oña Wilhelmi, E. 2011, in High-Energy Emission from Pulsars and their Systems, eds. D. F. Torres, \& N. Rea, 435

Dennerl, K., Haberl, F., Aschenbach, B., et al. 2001, A\&A, 365, L202

Dufton, P. L., Dunstall, P. R., Evans, C. J., et al. 2011, ApJ, 743, L22

Fryer, C. L. 1999, ApJ, 522, 413

Gast, H., Brun, F., Carrigan, S., et al. 2012 [arXiv: 1204 . 5860]

Gotthelf, E. V., \& Wang, Q. D. 1996, in Roentgenstrahlung from the Universe, eds. H. U. Zimmermann, J. Trümper, \& H. Yorke, 255

Heger, A., Woosley, S. E., \& Spruit, H. C. 2005, ApJ, 626, 350

Indebetouw, R., de Messières, G. E., Madden, S., et al. 2009, ApJ, 694, 84

Kardashev, N. S. 1962, AZh, 39, 393

Lattimer, J. M., \& Prakash, M. 2001, ApJ, 550, 426

Lazendic, J. S., Dickel, J. R., Haynes, R. F., Jones, P. A., \& White, G. L. 2000, ApJ, 540, 808

Li, T., \& Ma, Y. 1983, ApJ, 272, 317

Macri, L. M., Stanek, K. Z., Bersier, D., Greenhill, L. J., \& Reid, M. J. 2006, ApJ, 652, 1133

Manchester, R. N., Hobbs, G. B., Teoh, A., \& Hobbs, M. 2005, AJ, 129, 1993

Marshall, F. E., Gotthelf, E. V., Zhang, W., Middleditch, J., \& Wang, Q. D. 1998, ApJ, 499, L179

Marshall, F. E., Gotthelf, E. V., Middleditch, J., Wang, Q. D., \& Zhang, W. 2004, ApJ, 603, 682

Ott, C. D., Burrows, A., Thompson, T. A., Livne, E., \& Walder, R. 2006, ApJS, 164,130

Schöck, F. M., Büsching, I., de Jager, O. C., Eger, P., \& Vorster, M. J. 2010, A\&A, 515, A109

Venter, C., \& de Jager, O. C. 2007, in WE-Heraeus Seminar on Neutron Stars and Pulsars 40 years after the Discovery, eds. W. Becker, \& H. H. Huang, 40 Wang, Q. D., \& Gotthelf, E. V. 1998, ApJ, 494, 623

Wang, Q. D., Gotthelf, E. V., Chu, Y.-H., \& Dickel, J. R. 2001, ApJ, 559, 275

1 Universität Hamburg, Institut für Experimentalphysik, Luruper Chaussee 149, 22761 Hamburg, Germany

2 Laboratoire Univers et Particules de Montpellier, Université Montpellier 2, CNRS/IN2P3, CC 72, Place Eugène Bataillon, 34095 Montpellier Cedex 5, France

3 Max-Planck-Institut für Kernphysik, PO Box 103980, 69029 Heidelberg, Germany

4 Dublin Institute for Advanced Studies, 31 Fitzwilliam Place, Dublin 2, Ireland

5 National Academy of Sciences of the Republic of Armenia, Yerevan, Armenia

6 Yerevan Physics Institute, 2 Alikhanian Brothers St., 375036 Yerevan, Armenia
7 Universität Erlangen-Nürnberg, Physikalisches Institut, ErwinRommel-Str. 1, 91058 Erlangen, Germany

8 University of Durham, Department of Physics, South Road, Durham DH1 3LE, UK

9 Nicolaus Copernicus Astronomical Center, ul. Bartycka 18, 00-716 Warsaw, Poland

${ }^{10}$ CEA Saclay, DSM/IRFU, 91191 Gif-Sur-Yvette Cedex, France

11 APC, AstroParticule et Cosmologie, Université Paris Diderot, CNRS/IN2P3, CEA/Irfu, Observatoire de Paris, Sorbonne Paris Cité, 10 rue Alice Domon et Léonie Duquet, 75205 Paris Cedex 13, France

12 Laboratoire Leprince-Ringuet, Ecole Polytechnique, CNRS/IN2P3, 91128 Palaiseau, France

13 Institut für Theoretische Physik, Lehrstuhl IV: Weltraum und Astrophysik, Ruhr-Universität Bochum, 44780 Bochum, Germany

14 Institut für Physik, Humboldt-Universität zu Berlin, Newtonstr. 15, 12489 Berlin, Germany

15 LUTH, Observatoire de Paris, CNRS, Université Paris Diderot, 5 Place Jules Janssen, 92190 Meudon, France

16 LPNHE, Université Pierre et Marie Curie Paris 6, Université Denis Diderot Paris 7, CNRS/IN2P3, 4 Place Jussieu, 75252 Paris Cedex 5, France

17 Institut für Astronomie und Astrophysik, Universität Tübingen, Sand 1, 72076 Tübingen, Germany

18 Astronomical Observatory, The University of Warsaw, Al. Ujazdowskie 4, 00-478 Warsaw, Poland

19 Unit for Space Physics, North-West University, Potchefstroom 2520, South Africa

${ }^{20}$ Landessternwarte, Universität Heidelberg, Königstuhl, 69117 Heidelberg, Germany

21 Oskar Klein Centre, Department of Physics, Stockholm University, Albanova University Center, 10691 Stockholm, Sweden

22 Université Bordeaux 1, CNRS/IN2P3, Centre d'Études Nucléaires de Bordeaux Gradignan, 33175 Gradignan, France

${ }^{23}$ Funded by contract ERC-StG-259391 from the European Community

${ }^{24}$ University of Namibia, Department of Physics, Private Bag 13301, Windhoek, Namibia

${ }^{25}$ UJF-Grenoble 1 / CNRS-INSU, Institut de Planétologie et d'Astrophysique de Grenoble (IPAG) UMR 5274, 38041 Grenoble, France

26 Department of Physics and Astronomy, The University of Leicester, University Road, Leicester, LE1 7RH, UK

27 Instytut Fizyki Jạdrowej PAN, ul. Radzikowskiego 152, 31-342 Kraków, Poland

${ }^{28}$ Institut für Astro- und Teilchenphysik, Leopold-FranzensUniversität Innsbruck, 6020 Innsbruck, Austria

29 Laboratoire d'Annecy-le-Vieux de Physique des Particules, Université de Savoie, CNRS/IN2P3, 74941 Annecy-le-Vieux, France

e-mail: komin@lapp.in2p3.fr

30 Obserwatorium Astronomiczne, Uniwersytet Jagielloński, ul. Orla 171, 30-244 Kraków, Poland

31 Toruń Centre for Astronomy, Nicolaus Copernicus University, ul. Gagarina 11, 87-100 Torun, Poland

32 School of Chemistry \& Physics, University of Adelaide, Adelaide 5005, Australia

33 Charles University, Faculty of Mathematics and Physics, Institute of Particle and Nuclear Physics, V Holešovičkách 2, 18000 Prague 8, Czech Republic

34 School of Physics \& Astronomy, University of Leeds, Leeds LS2 9JT, UK 